\title{
MULTI-SCALE ROUGHNESS SPECTRA OF MOUNT ST. HELENS DEBRIS FLOWS
}

\author{
Richard T. Austin and Anthony W. England \\ Radiation Laboratory, Dept. of Electrical Engineering and Computer Science, University of Michigan
}

\begin{abstract}
A roughness spectrum allows surface structure to be interpreted as a sum of sinusoidal components with differing wavelengths. Knowledge of the roughness spectrum gives insight into the mechanisms responsible for electromagnetic scattering at a given wavelength. Measured spectra from 10-year-old primary debris flow surfaces at Mount St. Helens conform to a power-law spectral model, suggesting that these surfaces are scaling over the measured range of spatial frequencies. Measured spectra from water-deposited surfaces deviate from this model.
\end{abstract}

\section{Introduction}

Recent investigations [Evans et al., 1988; Gaddis et al., 1989] demonstrate that radar images are a useful tool in the study of volcanic terrain. Although there have been experimental investigations of radar scattering by such terrains, there have been few rigorous attempts to link the scattering cross section to quantitative surface roughness. Scattering from many volcanic terrains is difficult to model due to their extreme roughness and short correlation lengths. Rough surface scattering theories often assume that surfaces possess a Gaussian autocorrelation function (and corresponding Gaussian roughness spectrum) [Chen and Fung, 1988; Thorsos and Jackson, 1989]. In contrast, many natural surfaces are better described by power-law spectra [Berry and Hannay, 1978; Sayles and Thomas, 1978; Huang and Turcotte, 1989, 1990; England, 1992]. (These spectral forms are compared in Figure 1.) Rough surfaces that have structure over a wide range of spatial scales may be described using the concepts of fractal geometry introduced by Mandelbrot [1983]. Random rough fractal surfaces have power-law spectra; the slope of such a spectrum is related to the surface's fractal dimension (Austin et al., Special Problems in the Estimation of Power-Law Spectra as Applied to Topographical Modeling, submitted to IEEE Transactions on Geoscience and Remote Sensing, 1993, hereinafter referred to as Austin et al., submitted manuscript, 1993). In this report, we examine estimates of the roughness spectra of volcanic debris flows at several spatial scales.

\section{Debris Flow Measurements}

Surface roughness measurements were performed on several debris flows near Mount St. Helens (Cascade Range, northwestern United States) during September 1990. Mt. St. Helens was chosen because of its ease of access and its

\section{Copyright 1993 by the American Geophysical Union.}

extremely young terrains. The surfaces that we examined were located in the debris avalanche west-northwest of the volcano, along the North Fork Toutle River valley (Figure 2). Most of the debris was deposited during the eruption of Mount St. Helens on 18 May 1980. Since then, the deposits have undergone erosion by wind and water. The sites chosen for measurement were visually representative of principal surface textures, accessible to logging roads, and located in large homogeneous areas which should be distinguishable in a radar image. Figure 3 shows one of the rougher surfaces. A geologic description of the debris avalanche is given by Glicken [1989].

Our objectives were to characterize the surface roughness of the debris flows at scales smaller than, on the order of, and larger than the radar wavelength of common remote sensing radars. Two measurement techniques were used. A computer-driven, 2D laser profilometer recorded surface height profiles of square grids with sides between $8 \mathrm{~cm}$ and $1 \mathrm{~m}$ in length. The grids were sampled at intervals ranging from $2 \mathrm{~mm}$ to $2 \mathrm{~cm}$. We used surveying instruments to measure elevation at grid points within larger square areas measuring $32 \mathrm{~m}$ on a side. Sampling intervals within the larger squares were 1-4 m.

The 2D laser profilometer system was designed specifically for this experiment. Its main component is a surveying electronic distancemeter (EDM), which uses an infrared laser to measure distance. The EDM is mounted on an XY table, allowing the EDM to scan a surface area of up to $1 \mathrm{~m}^{2}$. The EDM laser has a spot diameter of approximately $1.5 \mathrm{~mm}$. In its most precise mode, the standard deviation of the measured surface height is $3 \mathrm{~mm}$. Surface height profiles from a typical grid are shown in Figure 4. While the two large rocks are quite prominent, a larger sample of the surface would show even larger "lumps."

Larger-scale topography was surveyed. Square grids with sides of $32 \mathrm{~m}$ were delineated by cables with markers at $1 \mathrm{~m}$ intervals. A self-leveling surveying level and stadia rod were then used to measure the surface height at intervals of 1-4 m, depending on the surface roughness.

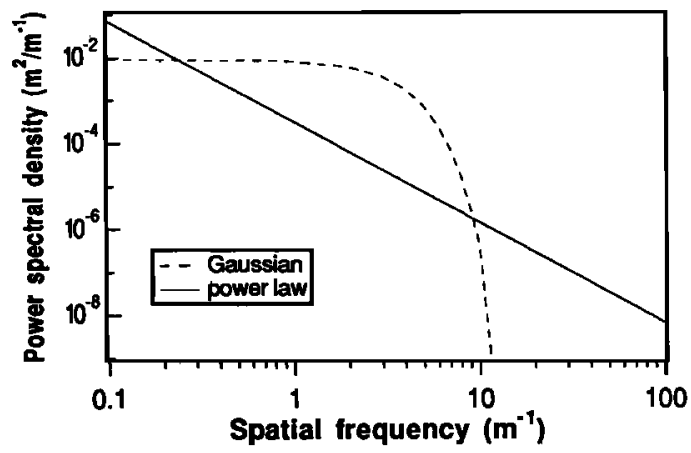

Fig. 1. A comparison of Gaussian and power-law spectra. 


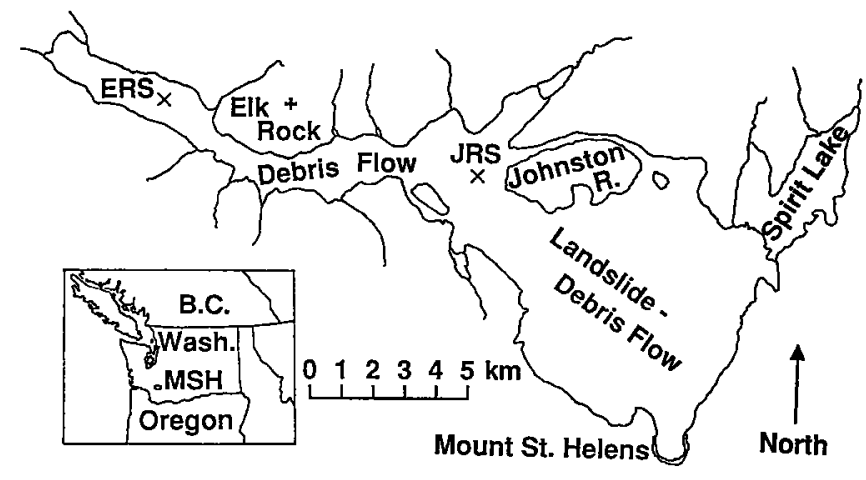

Fig. 2. The Johnston Ridge site (JRS) is located about $10 \mathrm{~km}$ northwest of the crater. The Elk Rock site (ERS) is located farther west, about $19 \mathrm{~km}$ west-northwest of the crater.

\section{Spectral Estimation}

Estimates of the roughness spectrum were calculated from both types of elevation data. Profilometer data suffered from errors which prevented the direct application of spectral estimation techniques. Since the measurements could not be repeated, it was necessary to apply corrective filtering. The procedure is fully described in Austin and England [1991].

As previously noted, investigators have found that many natural surfaces are scaling. Linear profiles of such surfaces have (over the range of measurable spatial frequencies) a surface roughness spectrum (power spectral density of surface height) $S_{Z}$ that has the form of a power law:

$$
S_{Z}(f)=c|f|^{-\beta},
$$

where $f$ is the spatial frequency and $c$ and $\beta$ are constants, with $1<\beta<3$. $\beta$ is sometimes called the spectral slope; a power-law spectrum is linear with slope $(-\beta)$ when plotted on a $\log -\log$ scale.

Power-law surfaces with isotropic statistics have a twodimensional roughness spectrum $S_{Z 2 D}$ that has a similar form:

$$
S_{Z 2 D}\left(f_{r}\right)=a f_{r}^{-\gamma},
$$

where $f_{r}$ is the radial spatial frequency satisfying $f_{r}^{2}=$ $f_{x}^{2}+f_{y}^{2}$, and $2<\gamma<4$. Because estimation of directional spectra requires a much greater quantity of data,

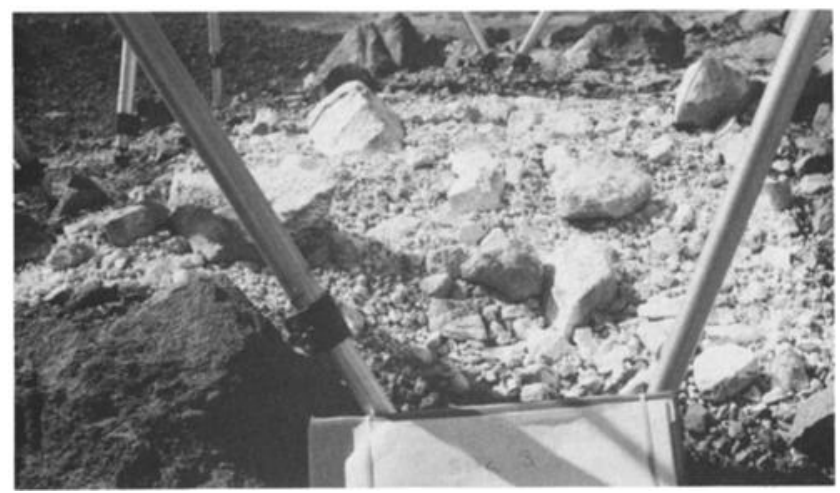

Fig. 3. A sample surface scanned by the EDM. The surface has been spray-painted to increase its reflectivity.

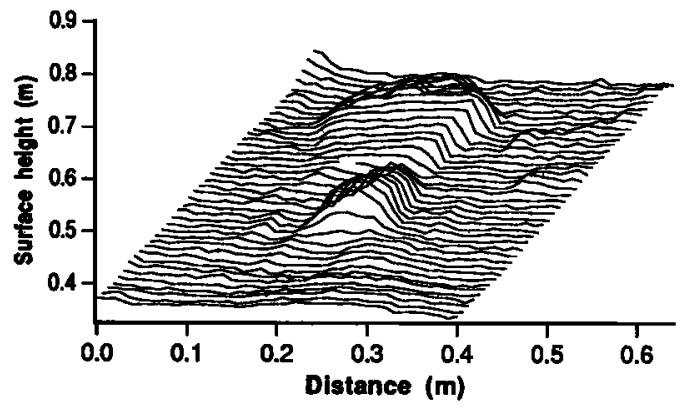

Fig. 4. A typical profilometer scan, showing surface height profiles. The grid measures $40 \mathrm{~cm} \times 40 \mathrm{~cm}$, with data points spaced $1 \mathrm{~cm}$ apart. Total surface height variation is $10.8 \mathrm{~cm}$.

we assume in this study that the surfaces have isotropic statistics as a first-order approximation.

Power-law spectra introduce unique difficulties in the spectral estimation process. In a related paper (Austin et al., submitted manuscript, 1993), we describe how Capon's estimator provides an estimate of the roughness spectrum that is better than that given by Fourier-based estimators (such as the periodogram) in the power-law case. Capon's estimator (described in Kay [1988]) essentially customizes a filter at each frequency of interest to minimize the total power output subject to the constraint that the gain at the frequency of interest is unity. In the power-law case, the low-frequency side lobes are reduced, thus Capon's estimator suffers less from spectral leakage, which can make Fourier estimates insensitive to the power-law slope $\beta$. Capon's estimator also has less variance than comparable Fourier-based estimators.

Capon's estimator, $\hat{P}_{C A P}(f)$, is obtained by first calculating an estimate of the autocorrelation matrix $\mathbf{R}_{Z Z}$, whose elements are defined as

$$
\left[\mathbf{R}_{Z Z}\right]_{k l}=E[Z[n] Z[n+k-l]],
$$

where $Z[n]$ is the surface height and $E[\cdot]$ indicates an expected value. Capon's estimator is then given by

$$
\hat{P}_{C A P}(f)=\frac{p \Delta}{\mathbf{e}^{H} \hat{\mathbf{R}}_{Z Z}^{-1} \mathbf{e}},
$$

where $\boldsymbol{p}$ is the maximum dimension of the autocorrelation matrix, $\Delta$ is the sampling interval, $\hat{\mathbf{R}}_{Z Z}^{-1}$ is the inverse of an estimate of the autocorrelation matrix, and

$$
\mathbf{e}=\left[\begin{array}{lllll}
1 & e^{j 2 \pi f \Delta} & e^{j 4 \pi f \Delta} & \cdots & e^{j 2 \pi(p-1) f \Delta}
\end{array}\right]^{T},
$$

in which $f$ is the spatial frequency. $\mathrm{e}^{H}$ is the Hermitian transpose of (5).

The bias of Capon's estimator is independent of $N$ (the number of points in a sampled row) but is dependent on $p$, the dimension of the autocorrelation matrix. For a data set of length $N$, an increase in $p$ will result in reduced bias at the cost of increased variance. We set $p \approx 0.3 N$ and calculated a Capon's spectral estimate for each row of each profilometer or survey grid. (The mean row height was subtracted before calculating the estimate.) The spectral estimates were then averaged over the rows of each grid. 


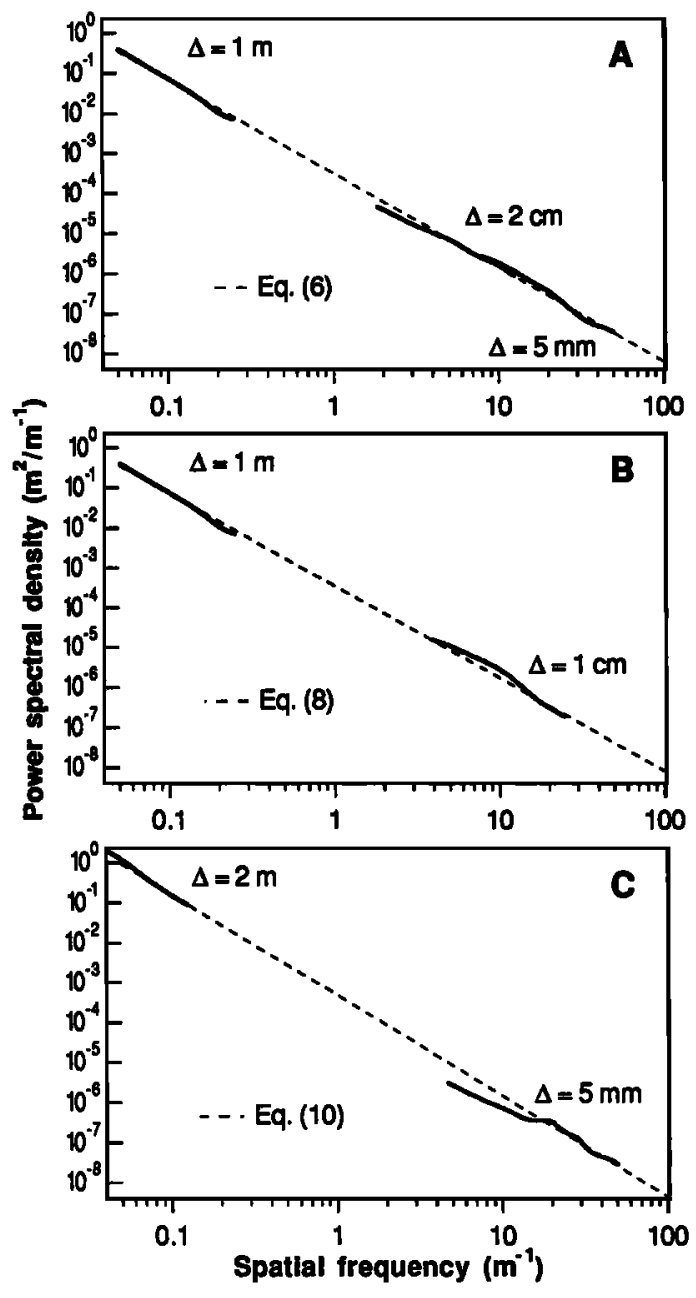

Fig. 5. (A) Survey and profilometer spectra from a JRS primary debris flow. (B) The same survey spectrum with a profilometer spectrum from an adjacent debris flow. (C) Spectra from a mixed terrain survey and a primary debris flow scan at ERS.

The high-frequency half of each averaged estimate was discarded to reduce the effect of aliasing. Averaged estimates at a single scale or at multiple scales were then fit with a power-law function (1), where appropriate, using a minimum absolute deviation fit. Profile (1D) spectra fitting the power-law model were then converted to surface (2D) spectra using the formulation given in (Austin et al., submitted manuscript, 1993), in which the 1D and 2D roughness spectra are related through their corresponding autocorrelation functions and the $2 \mathrm{D}$ autocorrelation function is assumed isotropic.

Although this method of determining the surface spectrum from linear profiles was made necessary by the profilometer problems, it is not without benefit. One grid of measured surface heights yields many linear profiles. The profile rows are not completely independent, but the row spectral estimates can still be averaged to obtain a significant decrease in variance, allowing us to set $p$ (in Capon's estimator) higher to decrease the bias. This averaging would not be possible with a two-dimensional estimator, given the same measured data.

\section{Measured Roughness Spectra}

The first site that we examined (JRS) was located in the debris flow area adjacent to and due west of Johnston Ridge (see Figure 2). This area of the debris flow exhibited large variations in relief (e.g., $8 \mathrm{~m}$ in a $32 \mathrm{~m} \times 32 \mathrm{~m}$ survey grid). The roughest surfaces that we measured in this area were the primary debris flow surfaces, i.e., debris flow surfaces whose present state is due to the removal of material by wind and water. Spectra from the roughest of these are shown in Figure 5A. The survey spectrum is the curve extending from 0.05 to $0.25 \mathrm{~m}^{-1}$; the higher frequency spectra were computed from profilometer scans. The linear character of these spectral estimates suggests that a power-law spectrum is appropriate for this surface. The equation of the power-law fit to these estimates is

$$
\hat{S}_{Z}(f)=\left(3.21 \times 10^{-4}\right)|f|^{-2.34} \text {. }
$$

(The hat denotes an estimate.) This fit is shown as a dashed line in Figure 5A. While we do not know that the spectrum follows this power law at the intermediate (unmeasured) spatial frequencies, we do think that it is significant that both the meter- and centimeter-scale spectra are well fit by the same power law.

We can convert (6) to an estimate of the surface (2D) spectrum, obtaining the following power law:

$$
\hat{S}_{Z 2 D}\left(f_{r}\right)=\left(1.77 \times 10^{-4}\right) f_{r}^{-3.34} \text {. }
$$

In Figure 5B, the same survey spectrum is shown together with a profilometer spectrum collected on a similar

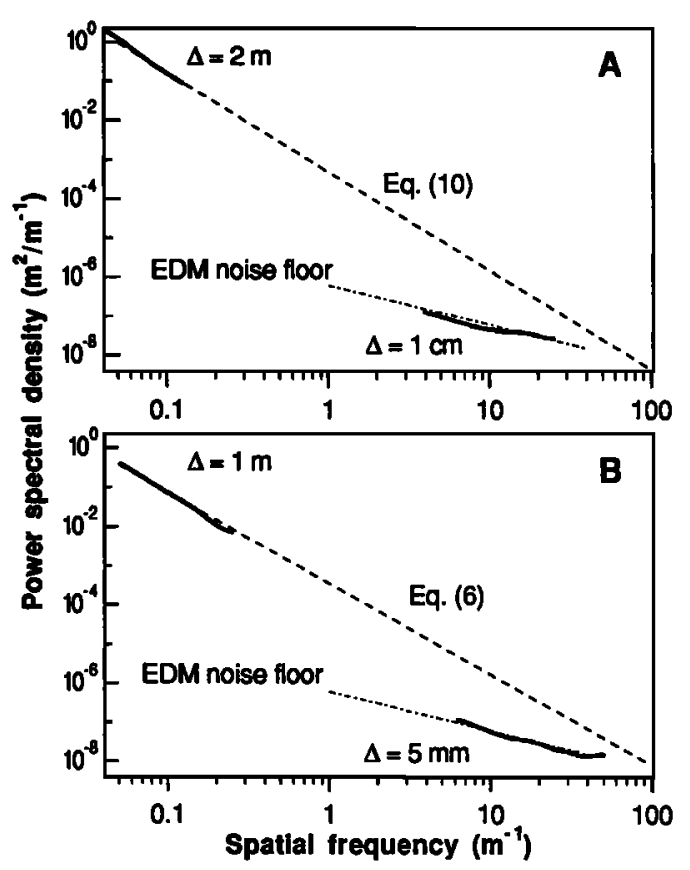

Fig. 6. (A) A profilometer spectrum $(\Delta=1 \mathrm{~cm})$ of an ERS sedimented surface, shown with the survey spectrum of the local mixed terrain, the power-law fit (10), and the EDM noise floor. (B) A profilometer spectrum $(\Delta=5 \mathrm{~mm})$ of a JRS sedimented surface, shown with the survey spectrum of the surrounding primary debris flow, the power-law fit (6), and the EDM noise floor. 
flow unit adjacent to the one surveyed. A power-law fit to these estimates gives the following spectra:

$$
\begin{aligned}
\hat{S}_{Z}(f) & =\left(3.57 \times 10^{-4}\right)|f|^{-2.31} \\
\hat{S}_{Z 2 D}\left(f_{r}\right) & =\left(1.95 \times 10^{-4}\right) f_{r}^{-3.31}
\end{aligned}
$$

The other area that we examined (ERS) was located in the North Fork Toutle River valley due west of Elk Rock. The valley was a mixture of hummocky debris mounds scattered across a flat, sedimented plain. A profilometer scan from one of the debris mounds yielded the spectral estimate shown at the high-frequency end of Figure 5C. The figure also shows an estimate from a survey grid containing both terrain types. (The debris mounds were typically smaller than the $32 \mathrm{~m} \times 32 \mathrm{~m}$ survey area.) A power-law fit may be appropriate here as well and is shown in the figure. The equations for this fit are

$$
\begin{aligned}
\hat{S}_{Z}(f) & =\left(4.98 \times 10^{-4}\right)|f|^{-2.51} \\
\hat{S}_{Z 2 D}\left(f_{r}\right) & =\left(2.86 \times 10^{-4}\right) f_{r}^{-3.51}
\end{aligned}
$$

Surfaces formed of water-deposited sediments within the hummocky debris had spectra that were markedly different from those of the primary surfaces. For example, in Figure $6 \mathrm{~A}$, we see that the spectral estimate from a scan of such a surface lies on the noise floor due to the limited precision of the EDM. The true spectrum of this surface must lie below this level and therefore cannot form a power-law trend with the survey spectrum of the local mixed terrain. This difference in spectral behavior is consistent with the visible character of the sedimented surface, which appeared quite flat. Similar remarks apply to the profilometer spectrum of a water-deposited sediment at the Johnston Ridge site, which is shown in Figure 6B with the survey spectrum of the surrounding primary debris flow.

\section{Conclusions}

Spectral estimates derived from multi-scale topographical measurements of several debris flow surfaces at Mount St. Helens show differences that correspond to observed surface characteristics. The extremely rough surfaces affiliated with 10-year-old primary debris flows possess roughness spectra that are well modeled by a power-law function at certain length scales, implying that these surfaces are scaling over the measured range of spatial frequencies. Surfaces created through sedimentation have spectra that lie below the noise floor at higher spatial frequencies and thus do not fit the power-law model.

While we are not yet able to develop a classifier of volcanic terrains based on surface roughness spectra, this study shows that a power-law spectrum is a reasonable descriptor of some volcanic surfaces. The scattering properties of such surfaces are not well understood. There has been some application of standard scattering models to surfaces of this type, but rougher volcanic terrains often violate assumptions made in these models (e.g., rms height $\ll$ radar wavelength). The present study and the others cited demonstrate the need for power-law scattering models in the remote sensing of volcanic debris flows and other natural surfaces. We are pursuing such a model through both theoretical and experimental studies.

Acknowledgments. The authors thank Bruce Babb and the staff of the Mount St. Helens National Volcanic Monument for their cooperation, Kyle McDonald and Bob Austin for assistance in the surface measurements, and two anonymous reviewers for their thoughtful comments and suggestions. This work is supported under NASA Grant NAGW-2199 and a shared NASA SIR-C project.

\section{References}

Austin, R. T., and A. W. England, Surface Characterization of Volcanic Debris Flows at Multiple Scales, in Proceedings of the 1991 International Geoscience and Remote Sensing Symposium, pp. 1675-1678, IEEE, New York, 1991.

Berry, M. V., and J. H. Hannay, Topography of random surfaces, Nature, 273, 573, 1978.

Chen, M. F., and A. K. Fung, A numerical study of the regions of validity of the Kirchhoff and small-perturbation rough surface scattering models, Radio Science, 23, 163-170, 1988.

England, A. W., The fractal dimension of diverse topographies and the effect of spatial windowing, in Geol. Soc. of Canada Paper 90-4: Ground Penetrating Radar, ed. J. A. Pilon, 57$61,1992$.

Evans, D. L., T. G. Farr, J. J. van Zyl, and H. A. Zebker, Radar Polarimetry: Analysis Tools and Applications, IEEE Trans. Geosci. Remote Sensing, 26, 774-789, 1988.

Gaddis, L., P. Mouginis-Mark, R. Singer, and V. Kaupp, Geologic analyses of Shuttle Imaging Radar (SIR-B) data of Kilauea Volcano, Hawaii, Geol. Soc. Am. Bull., 101, 317$332,1989$.

Glicken, H., Rockslide-debris avalanche of May 18, 1980, Mount St. Helens Volcano, Washington (USGS Prof. Paper 1488), 304 pp., 7 plates, 1989.

Huang, J., and D. L. Turcotte, Fractal Mapping of Digitized Images: Application to the Topography of Arizona and Comparisons With Synthetic Images, J. Geophys. Res., 94, 7491-7495, 1989.

Huang, J., and D. L. Turcotte, Fractal image analysis: application to the topography of Oregon and synthetic images, $J$. Opt. Soc. Am. A, 7, 1124-1130, 1990.

Mandelbrot, B. B., The Fractal Geometry of Nature, 468 pp., W. H. Freeman and Company, New York, 1983).

Sayles, R. S., and T. R. Thomas, Surface topography as a nonstationary random process, Nature, 271, 431-434, 1978.

Thorsos, E. I., and D. R. Jackson, The validity of the perturbation approximation for rough surface scattering using a Gaussian roughness spectrum, J. Acoust. Soc. Am., 86, 261-277, 1989.

R. T. Austin and A. W. England, Radiation Laboratory, 3228 EECS Bldg., University of Michigan, Ann Arbor, MI 48019-2122.

(Received February 11, 1993; revised May 14, 1993; accepted June 3, 1993.) 